Reprod. Nutr. Dévelop., 1981, 21 (2), 319-333.

\title{
Variations de la rétention azotée chez le Porc en croissance : conséquences sur le besoin
}

par Y. HENRY

Station de Recherches sur l'Elevage des Porcs, I.N.R.A., St-Gilles 35590 L'Hermitage.

Summary. Variations of protein retention in the growing pig : consequences on the protein requirement.

The first part of this paper deals with the distribution of the protein fraction (total protein, amino acids) in the tissues and body compartments in the growing pig, according to age, growth potential (sex, genotype) and nutritional factors (feeding level).

Overall protein retention is studied in the second part of the paper. The following points have been considered : changes in the protein retention curve during growth ; variations according to the type of pig and nutrient supply ; particular aspects of amino acid deposition with a relative constancy in the amino acid composition of deposited protein during the major part of growth.

In the third part, the following consequences of variations in protein retention on protein requirement for growth have been analyzed:

- relatively constant ratios between the requirements for individual essential amino acids and non-essential nitrogen (using lysine as a reference) ;

- relation between protein (amino acid) requirement and level of protein deposition; - variations in the efficiency of utilization of dietary amino acids for growth, according to muscle growth potential and production objectives (final slaughter weight, type of pig and feeding program).

The benefit of taking into consideration the components of apparent protein retention (synthesis and degradation) is discussed with a view to better adapting protein feeding to growth potential.

\section{Introduction.}

Les besoins en matières azotées et en acides aminés pour la croissance du Porc sont en relation avec l'importance du dépôt journalier de tissus maigres, ou encore avec les quantités journalières de protéines et d'acides aminés fixés en fonction de l'âge et/ou du poids vif. Cette intensité de développement de tissus maigres est ellemême fonction du potentiel de croissance des animaux (sexe, génotype) ou sous la dépendance du mode d'alimentation (alimentation à volonté ou restreinte) et d'élevage (pratique de la castration, poids d'abattage et type de carcasse).

L'étude des variations de la rétention azotée chez le Porc en croissance et de leurs conséquences sur la connaissance du besoin azoté nous conduit à examiner dans un 
premier temps les changements dans la distribution des tissus formés, ainsi que dans leur composition en protéines et en acilles aminés. Nous aborderons ensuite d'une manière plus globale les variations de la rétention azotée avant d'en dégager les conséquences au niveau des besoins en protéines et en acides aminés.

\section{I. - Variations de la répartition des tissus formés et de leur composition en protéines et en acides aminés.}

Les dépôts de protéines et d'acides aminés dans l'organisme sont la résultante des dépôts dans les différents compartiments corporels (muscle, os, graisses, peau et poils, sang, viscères et abats).

1. Protéines. - La répartition des protéines entre les compartiments corporels a été surtout étudiée à la faveur d'observations effectuées au poids commercial d'abattage (vers $100 \mathrm{~kg}$ ), selon un mode de fractionnement tenant compte des procédés habituels de découpe des carcasses. C'est ainsi que dans un Porc de $100 \mathrm{~kg}$ de poids vif, 80 à 85 p. 100 des protéines totales sont localisées dans la carcasse (tabl. 1). La fraction

\section{TABLEAU 1}

Distribution des protéines corporelles (p. 100) entre les différents compartiments de l'organisme chez le Porc selon le sexe (Just Nielsen, 1973)

\begin{tabular}{|c|c|c|}
\hline Sexe & Femelles & Mâles castrés \\
\hline 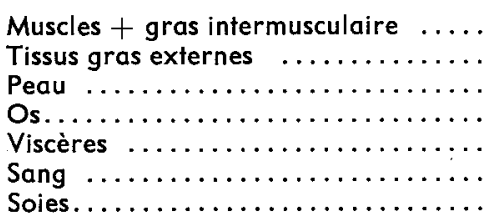 & $\begin{array}{r}55,3 \\
4,1 \\
9,3 \\
16,0 \\
8,4 \\
5,1 \\
1,8\end{array}$ & $\begin{array}{r}54,7 \\
4,5 \\
9,0 \\
16,3 \\
8,5 \\
5,1 \\
1,9\end{array}$ \\
\hline $\begin{array}{l}\text { Quantité totale de protéines }(\mathrm{kg}) \ldots \ldots \\
\text { Protéines ( } \mathrm{p} .100 \text { poids vif) } \ldots \ldots \ldots \ldots\end{array}$ & $\begin{array}{l}14,3 \\
17,5\end{array}$ & $\begin{array}{l}13,5 \\
16,9\end{array}$ \\
\hline
\end{tabular}

Porcs Landrace Danois de $90 \mathrm{~kg}$ de poids vif ; poids de carcasse $: 68,2 \mathrm{~kg}$.

consommable représente en moyenne 70 p. 100 du total, dont 50 à 55 p. 100 dans le tissu musculaire. Remarquons qu'une proportion imporiante des protéines (de l'ordre de 10 p. 100) est présente dans la peau. L'évolution avec l'âge (ou le poids vif) de la distribution des compartiments corporels au cours de la croissance du Porc se caractérise essentiellement par une diminution des proportions d'os, de viscères, abats et sang, au profit de la carcasse désossée. De la même façon, au fur et à mesure de la croissance, la part des protéines totales qui est présente dans l'os, dans les viscères ef les abats diminue, tandis que la proportion des protéines dans le muscle et le gras augmente, de même que celle du sang (tabl. 2). Comme l'indique le tableau 1 (Just 
TABLEAU 2

Evolution de la répartition des protéines corporelles (p.100) entre les différents compartiments de l'organisme chez le Porc en fonction du poids vif (Oslage, 1965)

\begin{tabular}{|c|c|c|c|c|c|}
\hline $\begin{array}{l}\text { Poids vif }(\mathrm{kg}) \quad \ldots \ldots \\
\text { Poids vif vide }(\mathrm{kg}) \ldots \ldots\end{array}$ & $\begin{array}{c}25 \\
22,7\end{array}$ & $\begin{array}{c}40 \\
36,6\end{array}$ & $\begin{array}{c}60 \\
55,5\end{array}$ & $\begin{array}{c}90 \\
84,3\end{array}$ & $\begin{array}{c}110 \\
103,4\end{array}$ \\
\hline 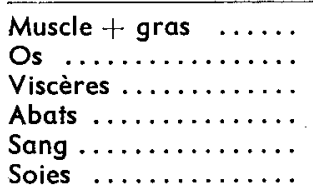 & $\begin{array}{r}64,7 \\
12,1 \\
8,9 \\
7,3 \\
4,9 \\
2,0\end{array}$ & $\begin{array}{r}67,6 \\
10,9 \\
8,8 \\
5,9 \\
4,8 \\
2,1\end{array}$ & $\begin{array}{r}71,2 \\
9,7 \\
7,2 \\
4,8 \\
5,9 \\
2,1\end{array}$ & $\begin{array}{r}72,5 \\
8,9 \\
6,8 \\
4,3 \\
5,3 \\
2,1\end{array}$ & $\begin{array}{r}72,4 \\
9,7 \\
6,1 \\
4,0 \\
5,8 \\
2,3\end{array}$ \\
\hline
\end{tabular}

Nielsen, 1973 ; Just Nielsen et Pedersen, 1975) la distribution des protéines dans l'organisme du Porc ne semble pas affectée par le sexe, quand on compare les femelles et les mâles castrés. En ce qui concerne le type génétique, on sait (Desmoulin, 1978) que la répartition des tissus dans l'organisme à un poids donné (rapporł muscle/graisse, rapport muscle/os) est plus ou moins fortement modifiée selon le potentiel de production de viande maigre, ce qui entraîne des variations élevées dans l'importance du dépôt azoté et sa distribution tissulaire et anatomique. La composition chimique des tissus eux-mêmes est affectée. Ainsi, le rapport protéines/lipides dans les muscles est plus élevé chez les types de Porcs à fort développement musculaire (Landrace Belge, Piétrain) comparativement à ceux présentant une conformation normale (Landrace Français). II s'ensuit que les différences enregistrées dans le développement des tissus maigres d'un type génétique à l'autre sont amplifiées lorsque l'on considère les protéines elles-mêmes, du fait précisément d'une contribution plus importante des dépôts gras intramusculaires chez les types de Porcs les plus gras.

Les facteurs nutritionnels peuvent également entraîner des modifications plus ou moins sensibles de la répartition des protéines corporelles. Ainsi, Metz et al. (1980) ont montré que pour une restriction de l'apport alimentaire de 20 p. 100 la quantité de protéines dans le poids vif vide diminue de 8 p. 100 , contre 5 p. 100 dans le muscle et 14 p. 100 dans le sang et les viscères, alors qu'elle demeure inchangée dans le compartiment osseux. Cette constatation présente un impact pratique dans la mesure où une réduction de l'apport énergétique de la ration contribue à un accroissement de la proportion de protéines consommables dans la carcasse.

2. Acides aminés. - La répartition des acides aminés entre les compartiments corporels résulte à la fois des variations de la distribution des protéines et de la composition de ces dernières en acides aminés. Ainsi, dans le cas particulier de la lysine, si l'on se réfère aux données de Wiesemüller et Poppe (1974), pour une quantité moyenne de $7,4 \mathrm{~g} / 16 \mathrm{~g} \mathrm{~N}$, les teneurs rencontrées dans les différentes fractions de l'organisme se situent entre $5,0 \mathrm{~g}$ dans l'os ef la peau ef $9,5 \mathrm{~g}$ dans les viscères. De même, Oslage et Schulz (1977) notent des écarts importants dans les teneurs des protéines en lysine et méthionine entre les différents tissus et organes. Quoi qu'il en soit, pendant la plus grande partie de la croissance, notamment au-delà de $25 \mathrm{~kg}$ de poids vif, ils n'observent pas d'évolution caractéristique du profil de composition en acides aminés des protéines corporelles (tabl. 3). Ceci nous conduit à conclure à une 
Evolution de la composition en acides aminés des protéines des compartiments corporels $(\mathrm{g} / 16 \mathrm{~g} \mathrm{~N}$ ) chez le Porc en fonction du poids vif (Oslage et Schulz, 1977)

\begin{tabular}{|c|c|c|c|c|}
\hline Poids vif $(\mathrm{kg}) \ldots \ldots \ldots \ldots \ldots$ & 24 & 53 & 81 & 112 \\
\hline \multicolumn{5}{|l|}{ Tissu musculaire : } \\
\hline Lysine ...... & 8,2 & 8,4 & 8,5 & 8,6 \\
\hline Méthionine & 2,4 & 2,6 & 2,8 & 2,9 \\
\hline Cystine .... & 1,2 & 1,2 & 1,4 & 1,3 \\
\hline \multicolumn{5}{|l|}{ Tissu adipeux: } \\
\hline Lysine $\ldots$ & 5,2 & 5,8 & 5,5 & 5,4 \\
\hline Méthionine & 1,4 & 1,4 & 1,6 & 1,7 \\
\hline Cystine .... & 1,0 & 1,3 & 1,0 & 1,2 \\
\hline \multicolumn{5}{|l|}{ Os : } \\
\hline Lysine & 4,3 & 4,5 & 4,0 & 3,8 \\
\hline Méthionine & 0,8 & 1,0 & 1,0 & 1,0 \\
\hline Cystine ... & 0,6 & 0,6 & 0,6 & 0,5 \\
\hline \multicolumn{5}{|l|}{ Peau + Oreilles + Poils } \\
\hline Lysine $\ldots \ldots \ldots \ldots \ldots \ldots \ldots$ & 4,5 & 4,4 & 4,2 & 4,2 \\
\hline Méthionine $\ldots \ldots \ldots \ldots \ldots$ & 1,1 & 1,0 & 1,0 & 1,2 \\
\hline Cystine $\ldots \ldots \ldots \ldots \ldots \ldots$ & 2,0 & 1,8 & 2,2 & 2,2 \\
\hline
\end{tabular}

certaine constance des teneurs en acides aminés de l'organisme au cours de l'engraissement, en dépit de légères variations dans la distribution de la fraction protéique des compartiments corporels. Il est à noter toutefois que pendant le jeune âge, entre la naissance et $20 \mathrm{~kg}$ de poids vif, les teneurs en acides aminés des protéines corporelles subissent des modifications relativement importantes, notamment au cours des premiers jours suivant la naissance (Aumaitre et Duée, 1974 ; Oslage et Schulz, 1977). Pendant cette période, on constate un accroissement des teneurs en certains acides aminés indispensables, en particulier en lysine, parallèlement à une diminution des concentrations en acides aminés non indispensables (hydroxyproline, glycine, proline).

\section{1. - Variations des dépôts de protéines et d'acides aminés.}

1. Evolution générale du dépôt protéique. - Les premières observations sur la rétention azotée chez le Porc en croissance, dans les conditions normales d'alimentation et d'élevage, ont fait apparaître une évolution de type curviliénaire avec l'âge ou le poids vif. Ainsi, selon Jespersen (1952) la quantité de protéines déposées quotidiennement augmentait de 50 à $60 \mathrm{~g}$ à $20 \mathrm{~kg}$ de poids vif jusqu'à un maximum de $100 \mathrm{~g}$ entre 60 ef $70 \mathrm{~kg}$ (soit entre 140 et 160 jours d'âge), correspondant à un gain pondéral de $800 \mathrm{~g} / \mathrm{jour}$, suivi d'une diminution progressive.

Au cours des travaux ultérieurs, les auteurs décrivent un autre type d'évolution de la rétention azotée. La valeur maximale de celle-ci est obłenue à une cadence plus rapide et plus précocement (aux alentours de $40 \mathrm{~kg}$ de poids vif), suivi d'un plateau prolongé jusqu'à $100 \mathrm{~kg}$ de poids vif (Hencken ef Freese, 1960). La même observation est faite par Oslage et al. (1966) avec des types de Porcs modernes sélectionnés pour la production de viande maigre. Dans une revue sur le sujet, Kielanowski (1972) 
précise que le dépôt journalier de protéines s'élève à $80 \mathrm{~g}$ dans les races de Porcs non améliorées, contre 100-110 g chez les Porcs conventionnels et environ $130 \mathrm{~g}$ chez les animaux de type maigre et à croissance rapide. Plus récemment, Whittemore (1977) note que le potentiel de rétention azotée chez le Porc se situe à un niveau constant pendant la plus grande partie de la période habituelle d'engraissement, soit entre 20 ef $120 \mathrm{~kg}$ de poids vif.

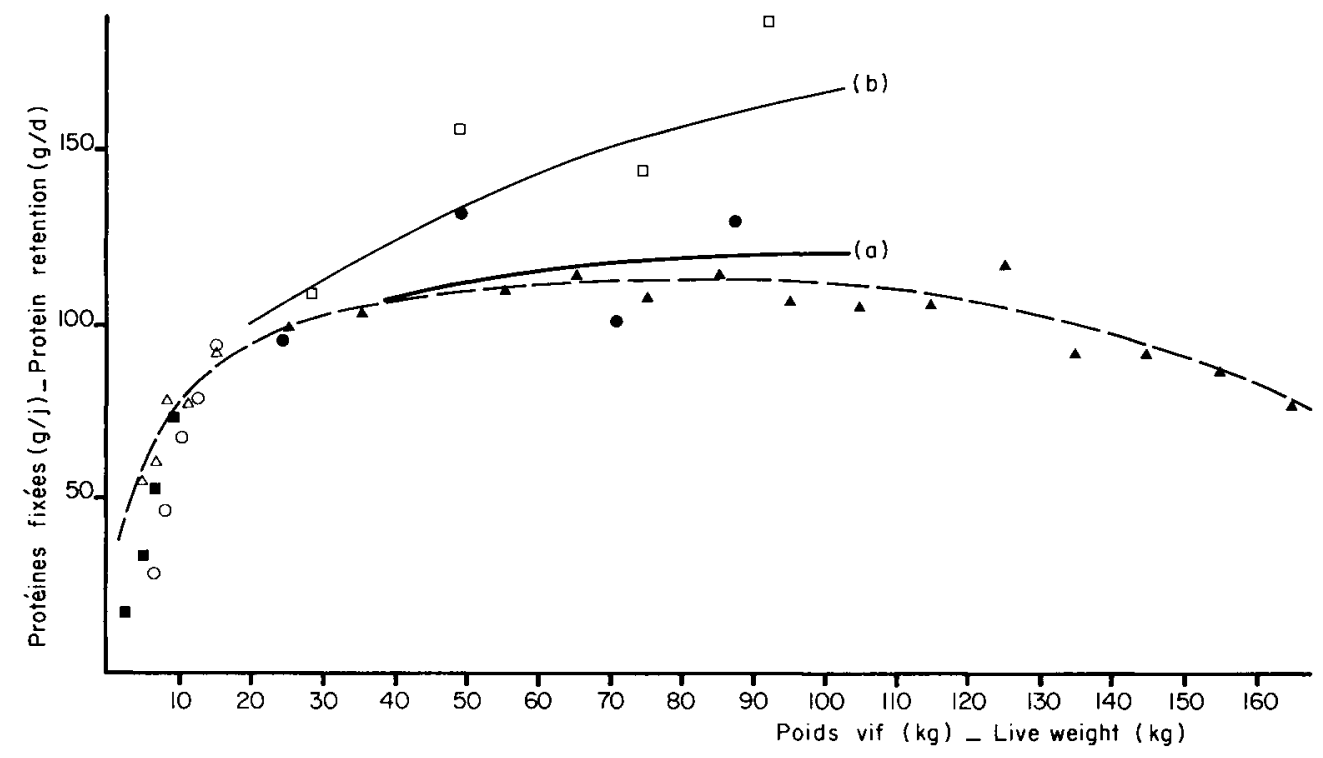

FIG. 1. - Rétention journalière de protéines chez le Porc en fonction du poids vif (données bibliographiques obtenues par la méthode des bilans) (Henry, Duée et Sève, 1978).

$\triangle$ : Rétention précoce maximale (Hencken ef Freese, 1960); $\circ,-$ : Porcelets sevrés respectivement à 3 semaines et 10 jours d'âge (Sève, non publié) ; 1 : Porcs Landrace allemands (Oslage ef al., 1966) ; $\square, \bullet:$ : Porcs Landrace Belge, respectivement mâles castrés $(a)$ et entiers (b) (Eeckhout, Bekaert ef Casteels, 1971).

Cette évolution dans le temps de la rétention azotée est le reflet d'une amélioration progressive du potentiel de croissance musculaire par la sélection dans le sens d'un développement corporel plus tardif et un poids à maturité plus élevé. Les types de Porcs à fort développement musculaire se distinguent même par un accroissement régulier du dépôt de protéines ou de tissus maigres jusqu'au poids habituel d'abattage, avec un retard consécutif dans l'apparition de la rétention maximale (Doornenbal, 1971 ; Desmoulin et Pommeret, 1975 ; Fowler, Bichard et Pease, 1976). L'évolution générale du dépôt azoté chez le Porc en fonction du poids vif est ainsi illustrée dans la figure 1, qui rassemble les données de la bibliographie (Henry, Duée et Sève, 1978).

Comme l'a indiqué Whittemore (1977) dans sa revue sur la prédiction des courbes de croissance chez le Porc, l'évolution du dépôt protéique cumulé au cours de la croissance est caractérisée respectivement par le contenu azoté final dans l'organisme à maturité physiologique ef l'âge auquel il est atteint. Suivant les valeurs de ces deux 
paramètres, différents types d'évolution du dépôt azoté sont ainsi observés. En particulier, un contenu azoté final plus élevé, pour un même âge à maturité, est obtenu avec une rétention azotée journalière (ou vitesse de dépôt de tissus maigres) plus forte, mais il est probable qu'un accroissement de l'intensité de développement musculaire ait alors pour conséquence une maturité physiologique retardée.

La modélisation de la capacité maximale de rétention azotée à partir des données expérimentales a fait l'objet de plusieurs tentatives. Les travaux de Gebhardt et coll. (Gebhardt et Müller, 1971 ; Gebhardt et al., 1973 ; Gebhardt, Ruhe et Koehler, 1978) ont montré la possibilité. d'un ajustement de la courbe de rétention azotée par unité de poids métabolique ( $\mathrm{N} \mathrm{g} /$ poids vif ${ }^{2 / 3}$, en $\mathrm{kg}$ ) en fonction du'niveau de l'apport azoté alimentaire ( $\mathrm{N}$ g/poids $\mathrm{vif}^{2 / 3}$, en $\mathrm{kg}$ ), selon une fonction exponentielle du type : $y=A\left(1-e^{-b x}\right)$ où $y$ et $x$ sont respectivement les quantifés d'azote fixé et ingéré par $\mathrm{kg}$ de poids $\mathrm{vif}^{2 / 3}$, et $\mathrm{A}$ la valeur asymptotique de la rétention azotée maximale. Pour des mâles castrés d'un poids vif compris entre 25 et $60 \mathrm{~kg}$, cette valeur s'établit à $2 \mathrm{~g} / \mathrm{kg}^{2 / 3}$.

Un même type de modélisation de la capacité de fixation des protéines corporelles a été proposé plus récemment par Menke (1979). Ceł auteur définit le potentiel de rétention azotée (A) comme la valeur maximale d'une fonction quadratique entre les quantités d'azote fixée et ingérée, exprimées en $\mathrm{kJ}$ par unité de poids métabolique (poids vif, en $\mathrm{kg}^{3 / 4}$ ). Ainsi, chez des Porcs de $30-40 \mathrm{~kg}$ de poids vif, cette valeur estimée de la capacité maximale de rétention azotée s'élève à $4,0 \mathrm{~g} \mathrm{~N} / \mathrm{kg}^{3 / 4}$, tandis que chez des Porcs de $80-100 \mathrm{~kg}$ elle n'est plus que de 1,0-1,3 g N/ kg $3 / 4$. Cela traduit une diminution du potentiel de rétention azotée avec l'accroissement du poids vif suivant une relation de type quadratique.

2. Facteurs de variation du dépôt azoté. - Le potentiel de rétention azotée, dans les conditions optimales d'alimentation et d'environnement, esł sous la dépendance des facteurs physiologiques (sexe, pratique de la castration) ef du type génétique. II convient par ailleurs de prendre en considération l'influence du niveau alimentaire en relation avec l'apport azoté (quantitatif et qualitatif) et la capacité de rétention azotée du type de Porc concerné.

2.1. Sexe et castration. - De nombreux travaux ont mis en évidence une capacité de rétention azotée plus élevée chez les mâles entiers que chez les femelles et a fortiori les mâles castrés. Dans une étude de Piatkowski et Jung (1966), les quantités moyennes journalières d'azote fixé dans l'intervalle de poids $30-110 \mathrm{~kg}$ sont ainsi respectivement de $21,2,18,4$ et $16,5 \mathrm{~g}$ dans les trois catégories d'animaux. La supériorité des mâles entiers sur les castrats pour fixer l'azote corporel, après une castration précoce (vers 2 semaines d'âge), est également confirmée par Desmoulin, Bonneau et Bourdon (1974), mais elle n'est effective que pendant la période de finition (à $70 \mathrm{~kg}$ de poids vif). Cela confirme les observations de Piatkowski ef Jung (1966), Fowler ef Livingstone (1972), concluant à une diminution plus précoce et plus rapide du dépôf journalier de tissus maigres chez les mâles castrés que chez les femelles. II convient de remarquer par ailleurs que l'effet défavorable de la castration est d'autant plus marqué que les animaux présentent une plus forte aptitude pour le développement de tissus maigres (Eeckhout, Bekaert et Casteels, 1971 ; Desmoulin, Bonneau et Bourdon, 1974). 
Il esł à noter que l'accroissement de la rétention azotée chez les Porcs mâles entiers comparativement aux mâles castrés est associé à une distribution différente de la fraction azotée dans les compartiments corporels. Le taux d'accroissement du dépôt azoté est notamment particulièrement important dans la peau : 30 p. 100 contre 10 p. 100 dans la fraction musculaire (Desmoulin, Bonnequ et Bourdon, 1974).

2.2. Génotype. - Les variations génétiques de la capacité ¿de rétention azotée s'expriment à la fois au niveau de l'intensité de la croissance pondérale et de la composition du croît (degré de musculature). C'est ce qui ressort d'une étude (Desloges, 1975) portant sur la comparaison de deux races à fort développement musculaire, l'une à croissance rapide (Landrace Belge), l'autre à croissance lente (Piétrain) par rapport à un type de Porc à conformation normale (Landrace Français). Avec une vitesse de croissance légèrement plus faible, les Porcs de type Landrace Belge présentent un dépôt azoté comparable à celui des animaux Landrace Français. Avec une vitesse de croissance plus lente, les animaux Piétrain, malgré l'hyperdéveloppement musculaire, ont une capacité de rétention azotée plus faible.

Les variations génétiques à l'intérieur d'une même race ont été décrites par McConnell, Barth et Griffin $(1971,1972)$. Les quantités d'azote fixées chez des Porcs dè type gras sont surtout réduites au cours de la phase de finition. Ceci confirme une fois de plus différentes observations montrant que c'est principalement pendant la phase terminale de l'engraissement que l'amélioration du potentiel génétique pour la production de viande maigre marque ses effets, par le maintien de la capacité de rétention azotée pendant une période prolongée de la croissance.

2.3. Niveau des apports alimentaires. - La rétention azotée chez le Porc est plus ou moins fortement influencée par le niveau de l'apport énergétique, à des degrés d'ailleurs variables selon l'intensité de la restriction, le stade de croissance considéré, le niveau de l'apport azoté et l'équilibre en acides aminés, enfin selon le potentiel de développement musculaire des animaux.

Dans une étude réalisée sur des jeunes Porcs femelles de race Large White entre 20 et $50 \mathrm{~kg}$ de poids vif, soumis à une restriction de l'apport énergétique journalier de 20 p. 100 par rapport à un niveau voisin du niveau à volonté (Rérat, Henry et Desmoulin, 1971), il est apparu que les performances optimales de croissance sont obłenues à l'aide d'un rapport matières azotées-énergie accru, quoique dans une proportion moindre que celle de la restriction énergétique. Cela signifie qu'une restriction énergétique relativement sévère appliquée à des animaux de type maigre (femelles) pendant la première phase de l'engraissement doit être associée à une compensation partielle de l'apport azoté journalier pour assurer le dépôt optimum d'azote dans l'organisme.

L'influence d'une restriction énergétique sur la rétention azotée selon le niveau de l'apport de l'acide aminé limitant dans le régime (lysine, chez le Porc à l'engraissement) a fait l'objet par ailleurs d'une série de travaux qui ont été résumés dans une revue de Wiesemüller (1980). Utilisant des Porcs mâles castrés dans l'intervalle de poids $40-120 \mathrm{~kg}$, l'auteur constate que les dépôts journaliers de protéines et de lysine ne sont pas affectés par une restriction alimentaire modérée (en moyenne 7 p. 100 d'un niveau voisin du niveau à volonté). Une réduction plus forte du niveau énergétique (en moyenne 14 p. 100 du niveau du témoin) entraîne par contre une diminution 
des dépôts de protéines et de lysine, parallèlement à la diminution du gain pondéral et du dépôt de gras. Toutefois, pendant la première partie de l'engraissement (entre 40 ef $70 \mathrm{~kg}$ de poids vif), le niveau énergétique le plus élevé procure une amélioration supplémentaire de la rétention azotée. Il résulte de ces observations que durant la phase initiale de la croissance, alors que la plus grande part de l'énergie est fixée sous forme de protéines, une réduction même légère de l'apport énergétique provoque une diminution à la fois du gain de tissus maigres et des dépôts gras. Cela rejoint les observations effectuées par Rérat et Desmoulin (1970) sur le Rat, dont la croissance se caractérise par un dépôt prédominant de tissus maigres. La même constatation a été faite chez le Porc en finition (Wiesemüller, 1980), lorsqu'à la faveur de l'amélioration génétique le potentiel de développement musculaire et de rétention azotée est maintenu à son niveau maximum au cours d'une période prolongée de la croissance. C'est ainsi qu'au-delà de $70 \mathrm{~kg}$ de poids vif, l'apport de lysine correspondant aux recommandations habituelles n'est plus suffisant pour exprimer la capacité maximale de rétention azotée chez les Porcs de type maigre, tandis qu'une réduction modérée du niveau énergétique entraîne de la même manière une diminution du dépôt journalier de tissus maigres. Cela confirme que l'interdépendance entre l'apport azoté ef l'apport énergétique est d'autant plus étroiłe que la capacité de production de viande maigre est accrue, notamment par voie génétique

En dehors de l'aspect quantitatif, la rétention azotée chez le Porc, pour un apport donné d'énergie métabolisable, peut être influencée par la nałure des substrats énergétiques, d'une façon semble-t-il différente suivant qu'il s'agit des activités de synthèse ou de catabolisme (Reeds et al., 1980).

Un autre point concerne les effets des apports alimentaires sur la rétention azotée selon le stade de croissance. Il en est ainsi notamment de la relation entre l'état nutritionnel pendant le jeune âge ef la croissance compensatrice ultérieure. De récentes observations (Sève, 1980 ; Le Dividich ef al., 1980) ont montré par exemple qu'en dépił d'une sous-consommation alimentaire pendant les jours suivant immédiatement un sevrage précoce (à 3 semaines) le porcelet continue à fixer de l'azote dans ses tissus grâce à une mobilisation momentanée des réserves lipidiques corporelles. A la suite des travaux de Wyllie et Owen (1978), Hogberg et Zimmerman (1978), il ressort par ailleurs qu'une restriction azotée de courte durée pendant le jeune âge peut provoquer une croissance compensatrice de tissus maigres au cours de la phase ultérieure d'engraissement, accompagnée d'une diminution des dépôts gras, dans les conditions d'alimentation selon l'appétit. En outre, selon Hogberg et Zimmerman, l'intensité de la réponse suivant une restriction azotée semble dépendre du potentiel de développement musculaire. Ainsi, chez les Porcs de type maigre, le phénomène de croissance compensatrice serait moins prononcé que chez les animaux de type gras au poids d'abattage de $100 \mathrm{~kg}$, pour autant qu'on puisse l'apprécier d'après le gain pondéral, l'indice de consommation et les caractéristiques de composition corporelle.

Le problème du déterminisme de la croissance compensatrice consécutive à une restriction azotée précoce et de courte durée n'en demeure pas moins posé. S'agit-il d'un changement dans le taux de renouvellement des protéines tissulaires, comme le laissent supposer les résultats obtenus sur rat par Millward et al. (1976) ? Une réduc- 
tion du catabolisme azoté pourrait entraîner un accroissement de l'anabolisme azoté apparent pendant la période de réalimentation, et par voie de conséquence une diminufion du besoin d'entretien et une augmentation de la part de l'énergie disponible pour la croissance. D'après Hogberg et Zimmerman (1979), les caractéristiques de développement des fibres musculaires (nombre de noyaux, diamètre de la fibre et poids du muscle) ne semblent pas modifiées au cours de cette période.

3. Dépôts d'acides aminés. - En raison de la relative constance de la composition des protéines corporelles, les dépôts d'acides aminés au cours de la croissance sonf surtout déterminés par le niveau de rétention azotée globale. Si l'on considère en particulier le compartiment musculaire, la composition de ses protéines en acides aminés n'est pas affectée par le sexe (Gruhn, 1965). De la même façon, elle est relativement peu modifiée par le type génétique. Dans une étude comparée de trois races de Porcs se différenciant par l'intensité de développement musculaire et la vitesse de croissance, Duée, Calmes et Desmoulin (1980) observent toutefois une augmentation sensible des teneurs en certains acides aminés indispensables (lysine, thréonine, méthionine) entre 40 et $60 \mathrm{~kg}$ de poids vif chez les Porcs de type Landrace Français et Piétrain, ef à un stade plus tardif (entre 60 et $80 \mathrm{~kg}$ ) chez les Porcs Landrace Belge. A partir des quantités de muscle mesurées par dissection (Desmoulin et Pommeret, 1975), ces auteurs constatent que les Porcs Landrace Belge déposent 10 p. 100 de lysine de plus que ceux de type Landrace Français, pour une vitesse de croissance comparable, entre 40 et $80 \mathrm{~kg}$ de poids vif, correspondant essentiellement à l'accroissement du dépôt de tissus maigres.

\section{III. - Conséquences sur le besoin azoté pour une croissance optimale.}

Comme il a été signalé par ailleurs (Henry, 1980a) et compte tenu de la part relativement faible de l'entretien dans le besoin azoté total (de l'ordre de 10 à 20 p. 100 en moyenne au cours de la croissance du Porc), les besoins en matières azotées et en acides aminés, normalement exprimés en quantités journalières, sont avant tout déterminés par l'importance des dépôts ainsi que par le rendement de fixation des quantités de matières azotées ou d'acides aminés absorbées. Les variations du besoin azoté, lorsqu'il est exprimé par rapport à l'énergie disponible, sont encore amplifiées, en raison de la relation inverse entre le niveau de l'apport énergétique requis et le potentiel de rétention azotée, pour une vitesse de croissance donnée (Henry et Etienne, 1978).

1. Relation entre les besoins en acides aminés indispensables ef en azote indifférencié. - Les faibles variations de la composition en acides aminés des protéines déposées au cours de la croissance, hormis le cas du tout jeune porcelet, ont permis d'établir l'hypothèse d'une relative constance des rapports entre les besoins en acides aminés indispensables pris individuellement ainsi que relativement au besoin azoté indifférencié. Ceci a conduit au concept d'une «protéine idéale» pour la croissance indépendamment des facteurs susceptibles de la modifier, tels que le sexe ou le type génétique (Williams et al., 1954 ; Fuller, 1978 ; Cole, 1978). C'est ainsi que les recommandations établies dans le cadre du NRC (1979) pour les différentes phases de la 
croissance ont été fixées par extrapolation linéaire à partir de l'intervalle de poids 20$35 \mathrm{~kg}$, les rapports entre les besoins en chacun des acides aminés ef celui en lysine éfant maintenus constants. De la même façon, le besoin en lysine entre 20 et $100 \mathrm{~kg}$ de poids vif représente une proportion sensiblement constante $(5,5$ p. 100 du besoin minimal en prołéines indifférenciées, une fois couverts les besoins en acides aminés indispensables (Henry, 1980b). Le tableau 4 donne une illustration des valeurs rela-

TABLEAU 4

Rapporfs entre les besoins en acides aminés indispensables en comparaison avec les teneurs relatives des proféines corporelles en acides aminés (par référence à la lysine : base 100)

\begin{tabular}{|c|c|c|}
\hline & Besoins ( ${ }^{1}$ ) & $\begin{array}{l}\text { Acides aminés } \\
\text { des protéines } \\
\text { corporelles }\left({ }^{2}\right)\end{array}$ \\
\hline 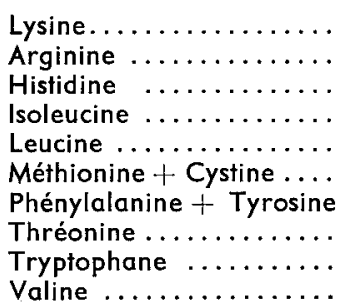 & $\begin{array}{r}100 \\
29 \\
26 \\
60 \\
72 \\
60 \\
100 \\
60 \\
20 \\
70\end{array}$ & $\begin{array}{r}100 \\
93 \\
42 \\
53 \\
106 \\
47 \\
92 \\
54 \\
63\end{array}$ \\
\hline
\end{tabular}

(1) Henry $(1980 a) ;\left({ }^{2}\right)$ Oslage et Schulz (1977).

tives des besoins en acides aminés indispensables (par référence à la lysine) en relation avec les valeurs correspondantes des teneurs en acides aminés des protéines corporelles.

Il convient cependant de considérer avec prudence ce mode d'établissement des recommandations azotées, basé sur l'existence de rapports constants entre les besoins des différents acides aminés. On sait, en effet, qu'il existe des fluctuations plus ou moins importantes dans la distribution de la fraction azotée dans les compartiments corporels, de même que dans les vitesses de synthèse et de dégradation des protéines à l'intérieur de ces compartiments (Millward ef al., 1976). Ainsi, chez les Porcelets d'un poids vif inférieur à $10 \mathrm{~kg}$, le besoin mesuré en lysine esł plus élevé que celui estimé par extrapolation suivant les indications du NRC (1979). D'autre part, selon Lewis ef al. (1977), le rapport tryptophane/lysine chez des porcelets de 5 à $10 \mathrm{~kg}$ semble également plus élevé que celui observé chez les animaux de 20 à $35 \mathrm{~kg}$ de poids vif. Cette différence peut être attribuée au moins en partie à une concentration plus importante des protéines corporelles en acides aminés indispensables au cours de la phase initiale de la croissance.

2. Relation besoin-dépôt. - A partir de la connaissance du dépôt azoté, parallèlement aux dépenses endogènes, diverses tentatives ont été faites pour estimer, par une démarche factorielle, le besoin azoté net pour la croissance (Kielanowski, 1972 ; Buraczewski, 1972 ; Oslage ef Schulz, 1977) ou encore le besoin total, compte tenu du rendement de fixation des acides aminés ef de l'azole ingéré (Wiesemüller, 1976a). 
En réalité, en raison de l'insuffisance des données disponibles sur les différentes composantes du besoin, il s'est avéré plus commode de moduler les apports recommandés en fonction du niveau des performances. C'est ainsi que l'on peut imaginer une correction du besoin azoté tenant compte du gain pondéral et des teneurs de ce dernier en protéines et en acides aminés aux différents stades de la croissance, ou encore en fonction du gain moyen et des caractéristiques de composition corporelle (rapport muscle ou tissus maigres/graisses) pendant la période considérée. Une telle correction a été proposée par Wiesemüller (1976b), mais sur la base d'une composition constante du croit au cours de l'engraissement. Avant d'aller plus avant dans cette démarche, qui n'en est pas moins nécessaire, il importe que l'on dispose d'informations suffisamment nombreuses et précises sur l'évolution de la croissance tissulaire chez le porc en fonction des capacités génétiques et physiologiques.

3. Rendement de l'utilisation des acides aminés pour la croissance. - On dispose de très peu de données sur le rendement de l'utilisation des acides aminés indispensables pour la croissance. D'après les travaux de Wiesemüller (1980), le taux moyen de conversion de la lysine alimentaire en lysine fixée au cours de la période d'engraissement (40 à $120 \mathrm{~kg}$ de poids vif), lorsque cet acide aminé constitue le facteur limitant du régime, s'élèverait à 40 p. 100. Selon le même auteur, la valeur maximale du rendement d'utilisation de la lysine était de $67 \mathrm{p}$. $100 \mathrm{chez}$ des femelles entre 40 et $70 \mathrm{~kg}$ de poids vif. Compte tenu de la disponibilité de la lysine, ces valeurs sont comparables à celles obtenues pour la rétention azotée avec des régimes équilibrés à la fois en acides aminés indispensables et en azote indifférencié.

Bien entendu, le potentiel de dépôt journalier de protéines ou de tissus maigres est la résultante des processus de synthèse et de catabolisme, qui sont probablement influencés de manière différente par les facteurs génétiques et physiologiques. Le problème est alors de savoir dans quelle mesure les variations observées dans le taux de renouvellement des protéines corporelles, selon leur localisation tissulaire et/ou anatomique, peuvent modifier l'importance des besoins en protéines et en acides aminés, en relation avec le potentiel de croissance des animaux. Comme le laisse apparaître une première étude exploratoire de Maner et al. (1977), on peut notamment s'interroger sur la possibilité de sélectionner, avec des régimes à faible taux de matières azotées, des porcs présentant des besoins abaissés en protéines et en acides aminés, tout en conservant leur potentiel de croissance et de développement musculaire. En clair, peut-on imaginer de tirer parti de la variabilifé génétique (si elle existe) dans les vitesses de synthèse et de dégradation des protéines tissulaires pour exploiter des animaux plus économes en protéines alimentaires, c'est-à-dire à bas niveau de catabolisme azoté ? Ou bien, au contraire, les vitesses de synthèse et de catabolisme, notamment pour les hauts niveaux de production, sont-elles indissolublement liées, comme on est tenté de le supposer d'après les premières observations sur ce sujet? Par ailleurs, il y a lieu de se demander dans quelle mesure l'importance croissante prise par le compartiment musculaire dans le pool azoté total, au fur ef à mesure de l'amélioration de la capacité de production de viande maigre, peut influer sur le besoin azoté, par le biais des activités de synthèse ef dégradation des protéines dans les différents compartiments corporels. Sur un plan plus général, le problème se pose dans les mêmes termes lors de l'adaptation du profil de l'apport azoté alimentaire 
tout au long de la croissance en fonction des objectifs de production (poids d'abattage, type de carcasse), suivant que l'on envisage par exemple la production d'animaux à fortes potentialités de croissance et nourris intensivement jusqu'au poids habituel d'abattage, ou la production d'animaux plus lourds d'un type génétique approprié (à définir) et soumis à une alimentation moins riche mais moins coûteuse.

\section{Conclusion.}

Les variations de la rétention azotée chez le Porc en croissance résultent d'un ensemble de processus qui intègrent l'évolution avec l'âge de la composition des tissus formés et de leur répartition entre les différents compartiments de l'organisme, suivant des modalités variables selon l'influence de divers facteurs liés notamment à l'animal (facteurs physiologiques, type génétique) ou à l'alimentation (apports alimentaires quantitatifs et qualitatifs). Les dépôts d'acides aminés sont avant tout déterminés par l'importance du dépôt azoté lui-même, en raison de la relative constance de la composition des protéines déposées en acides aminés.

On en déduit une relative constance dans les rapports entre les différents besoins en acides aminés indispensables et en azote indifférencié au cours de la plus grande partie de la croissance, ce qui introduit une certaine cohérence dans l'établissement des normes alimentaires. Les variations de la distribution de la fraction azotée entre les tissus et compartiments corporels, jointes aux différences d'intensité de synthèse et de dégradation des protéines formées d'un compartiment à l'autre, peuvent entraîner par ailleurs des modifications plus ou moins marquées dans le rendement de la fixation des protéines et acides aminés ingérés, et par voie de conséquence dans les modalités d'adéquation des apports alimentaires aux besoins spécifiques des animaux, selon leur potentiel de croissance et les objectifs de production.

En définitive, une plus grande maîtrise des facteurs de variation du besoin azoté chez le Porc implique une meilleure connaissance des variations du dépôt azoté et de sa localisation anatomique. Sur le plan expérimental, ceci conduit à la nécessité de compléter les mesures de bilan azoté global par une appréciation quantitative du dépôt journalier de tissus maigres et de sa distribution dans l'organisme mais avant tout dans la fraction noble, voire consommable, de la carcasse, en vue d'une transformation optimale des protéines par le porc pour les besoins de la consommation.

L'étude de la relation entre le besoin azoté et les variations du dépôt apparent de protéines et d'acides aminés constitue une étape décisive dans la modulation des normes d'apports alimentaires en fonction des facteurs de production. Mais pour aller au-delà dans l'adéquation des conditions de nutrition azotée du porc en croissance, il convient de prendre en considération les composantes du dépôt protéique apparent (synthèse, catabolisme), à l'aide de critères appropriés dans l'expérimentation sur des lots relativement importants d'animaux. 


\section{Références}

AUMAITRE A., DUÉE P. H., 1974. Composition en acides aminés des protéines corporelles du porcelet entre la naissance et l'âge de huit semaines. Ann. Zootech., 23, 231-236.

BURACZEWSKI S., 1972. Views on the pig's requirements for amino acids and new sources of these nutrients suitable for use in the feeding of pigs. Symp. New Developpements in the provision of amino acids in the diets of pigs and poultry. FAO, Econ. Soc. Counc., EEC, Geneva.

COLE D. J. A., 1978. Amino acid nutrition of the pig, 59-72. In HARESIGN W., LEWIS D., Recent advances in animal nutrition, Butterworths, London.

DESLOGES L., 1975. Interoction nutrifion énergétique et azotée et type génétique chez le porc. Mém. Fin Etudes, ENSA Rennes.

DESMOULIN B., 1978. Etudes sur la composition corporelle du porc. Applications scientifiques ou techniques. Journ. Rech. Porcine en France, 10, 211-234, INRA-ITP éd., Paris.

DESMOULIN B., POMMERET P., 1975. Evolution de la composition anatomique des porcs femelles des types Landrace Français, Landrace Belge et Piétrain : Conséquences sur les méthodes d'orientation des productions. Journ. Rech. Porcine en France, 7, 179-194, INRA-ITP éd., Paris.

DESMOULIN B., BONNEAU M., BOURDON D., 1974. Etude en bilan azoté et composition corporelle des porcs mâles entiers ou castrés de race Large White. Journ. Rech. Porcine en France, 7, 247255, INRA-ITP éd., Paris.

DOORNENBAL H., 1971. Growth, development and chemical composition of the pig. I. Lean tissue and protein. Growth, 35, 281-295.

DUÉE P. H., CALMES R., DESMOULIN B., 1980. Composition en acides aminés des protéines musculaires du pore selon le type génétique. Ann. Zootech., 29, 31-37.

EECKHOUT W., BEKAERT H., CASTEELS M., 1971. Essais comparatifs de digestibilité et de bilans azotés chez les verrats et les mâles châtrés de race Porc Belge. Rev. Agric., 24, 41-58.

FOWLER V. R., BICHARD M., PEASE A., 1976. Objectives in pig breeding. Anim. Prod., 23, 365-387.

FOWLER V. R., LIVINGSTONE R. N., 1972. Modern concepts of growth in pigs, 143-161. In COLE D. J. A., Pig production, Butterworths, London.

FULLER M. F., 1978. Amino acids in the nutrition of the pig. Rowett Res. Inst., Annu. Rep. Stud. anim. Nutr. allied Sci., 34, 116-128.

GEBHARDT G., MÜLLER H., 1971. Bestimmung des Maximalen N-Retentions vermögens wachsender Schweine von $25-60 \mathrm{~kg}$. Arch. Tierernähr. 21, 183-192.

GEBHARDT G., MÜLLER H., FRENZEL F., HIDALGO A., 1973. Das Maximale N-Retentionsvermögen wachsender Schweine in Abhängigkeit von der Lebendmasse. Wiss. Z. Karl-Marx-Univ. Leipzig. Math. Naturwiss. Reihe, 22, 225-233.

GEBHARDT G., RUHE Chr., KOEHLER R., 1978. Biologische Grundlagen des Wachstums unter besonderer Berucksichtigung von N-Stoffwechselprozessen der Monogastriden. Akad. Landwitsch. D. D. R. (170), 5-29.

GRUHN K., 1965. Einflusz des Alters auf Nährstoffgehalt und Fleischeiweisszusammensetzung bei Schweinen. Die Nährung, 9, 325-333.

HENCKEN H., FREESE H. H., 1960. Gezielte Fütterung auf Fleischansatz beim Schwein. Scheinezuch Scheinemast. (8), 61-63.

HENRY Y., 1980a. Protein and amino acid requirements of growing pigs. 3rd Sym. Protein metabolism and nutrition, EAAP, Braunschweig, 634-657.

HENRY Y., 1980b. Besoin azoté global du porc en croissance : résultats sur femelles ef synthèse. journ. Rech. Porcine en France, 12, 183-194, INRA-ITP éd., Paris.

HENRY Y., ETIENNE M., 1978. Alimentation énergétique du porc. Journ. Rech. Porcine en France, 10, 119-166, INRA-ITP éd., Paris.

HENRY Y., DUÉE P. H., SĖVE B., 1978. Construction of the amino acid requirement of the pig 29th annu. Meet. EAAP. Commission on animal Nutrition, Stockholm.

HOGBERG M. G., ZIMMERMAN D. R., 1978. Compensatory responses to dietary protein, length of starter period and strain of pig. J. anim. Sci., 47, 893-899.

HOGBERG M. G., ZIMMERMAN D. R., 1979. Effects of protein nutrition in young pigs of developmental changes in the body and skeletal muscles during growth. J. anim. Sci., 49, 472-481. 
JESPERSEN J., 1952. Übersicht über die Arbeit der Staatlichen Tierzuchtanstelt in Jahre 1951-52. II. Abteilung für Schweineversuche. Züchtungskunde, 24, 229-230.

JUST NIELSEN A., 1973. Anatomical and chemical composition of danish Landrace pigs slaughtered at $90 \mathrm{~kg}$ live weight in relation to litter, sex and feed composition. J. anim. Sci., 36, 476-483.

JUST NIELSEN A., PEDERSEN O. K., 1975. Danish investigations concerning body composition of pigs in relation to nutrition, sex and slaughter weight. 26th annu. Meet. EAAP, Commission on Pig Production, Warsaw.

KIELANOWSKI J., 1972. Protein requirements of growing animals, 528-546. In LENKEIT W., BREIREM K., CRASEMANN E., Handbuch für Tierenährung, vol. 2. Paul Parey, Hamburg.

LE DIVIDICH J., VERMOREL M., NOBLET J., BOUVIER J. C., AUMAITRE A., 1980. Effects of environmental temperature on heat production, energy retention protein and fat gain in early weaned piglets. Brit. J. Nutr., 44, 313-323.

LEWIS A. J., PEO E. R., Jr., CUNNINGHAM P. J., MOSER B. D., 1977. Determination of the optimum dietary proportions of lysine and tryptophan for growing pigs based on growth, food intake and plasma metabolites. J. Nutr., 107, 1369-1376.

MANER J. H., ROUNSAVILLE T. R., GALLO J. T., POND W. G., VAN VLECK L. D., 1977. Genetic differences in protein requirement of growing swine. J. onim. Sci., 45, 1243-1246.

Mc CONNELL J. C., BARTH K. M., GRIFFIN S. A., 1971. Nutrient digestibility and nitrogen metabolism studies at different stages of growth with fat and lean type swine fed two levels of protein. J. anim. Sci., 32, 654-657.

Mc CONNELL J. C., BARTH K. M., GRIFFIN S. A., 1972. Nitrogen metabolism at three stages of development and its relationship to measurements of carcass composition in fat and lean type swine. J. anim. Sci., 35, 556-560.

MENKE K. H., 1979. Ein Vorschlag zur Definition und zur experimentellen Bestimmung des Proteinretentionsvermögens wachsender Tiere. Z. Tierphysiol. Tierernähr. Futtermittelkde, 42, 173-189.

METZ S. H. M., BERGSTRÖM R. L., LENIS N. P., de WIJS M., DEKKER R. A., 1980. The effect of daily energy intake on growth rate and composition of weight gain in pigs. Livest. Prod. Sci., 7, 79-87.

MILLWARD D. J., GARLICK P. J., JAMES W. P. T., SENDER P. M., WATERLOW J. C., 1976. Protein turnover, 49-69. In COLE D. J. A., BOORMAN K. N., BUTTERY P. J., LEWIS D., NEALE R. J., SWAN H., Protein metabolism and nutrition, EAAP Publ, no 16, Butterworths, London.

N.R.C. (National Research Council), 1979. Nutrient requirements of swine, Publ. nat. Acad. Sci., NRC, Washington D. C.

OSLAGE H. J., 1965. N (Eiweisz)- und Fett verteilung im körper wachsender Schweine. Züchtungskunde, 37, 339-347.

OSLAGE H. J., FLIEGEL H., FARRIES F. E., RICHTER K., 1966. Stickstoff-, Fett- und Energieansatz bei Wachsenden Mastchweinen. Z. Tierphysiol. Tierernähr. Fuffermitfelkde, 21, 50-65.

OSLAGE H. J., SCHULZ E., 1977. Aminosäurenansatz in verschiedenen Organen und im Gesamtkörper wachsender Schweine sowie Ableitungen zum Aminosäurenbedarf. Vth Int. Symp. Amino Acids, Budapest.

PIATKOWSKI B., JUNG H., 1966. Der Eiweissansatz wachsender Schweine verschiedenen Geschlechts und Verschiedener Typrichtung. I. Stickstoffansatz und Verwertung sowie Gewichtszuwachs in den einzelnen Wachtstumabschnitten. Arch. Tierz., 9, 307-319.

REEDS P. J., FULLER M. F., CADENHEAD A., LOBLEY G. E., 1980. The effects of diefary energy and protein on protein turnover and nitrogen balance in growing pigs. 3rd Symp. Protein metabolism and nutrition, EAAP. Braunschweig, 67-74.

RÉRAT A., DESMOULIN B., 1970. Influence d'une restriction alimentaire, énergétique et azotée sur la croissance et la composition corporelle du rat blanc. Ann. Zootech., 19, 103-115.

RÉRAT A., HENRY Y., DESMOULIN B., 1971. Influence d'une restriction énergétique sur le besoin azoté du porc femelle. Journ. Rech. Porcine en France, 3, 65-72, INRA-ITP éd., Paris.

SÈVE B., 1980. Le rationnement du porcelet après sevrage : interaction avec l'apport de matières azotées. Journ. Rech. Porcine en France, 12, 195-202, INRA-ITP éd., Paris.

WHITTEMORE C. T., 1977. The prediction of growth responses in pigs, 158-166. In HARESIGN W., LEWIS D., Recent advances in animal nutrition. Butterworths, London.

WIESEMÜLLER W., 1976a. Aminosäurenbedarf und -Bedarfsdeckung bei Schweinen. Wisserıschaft. Zeitschr. Wilhem-Pieck. Univ., Rosiock, 2, 151-164.

WIESEMÜLLER W., 1976b. Prinzipien der Protein- und Aminosäurenbedarfermittlung bei Matschweinen. Tag-Ber., Akad. Landwirtsch. Wiss. DDR, Berlin, 142, 85-90. 
WIESEMÜLLER W., 1980. Amino acid requirement of the pig: methods of approach and recommendations. Ann. Zootech., 29, 179-193.

WIESEMÜLLER W., POPPE S., 1974. Untersuchungen zum Aminosäurenbedarf von Mastchweinen (Börge). 5. - Einflusz der Aminosäurensernährung auf den Proteinansatz. Arch. Tierernähr., 24, 535-542.

WILLIAMS H. H., CURTIN L. V., ABRAHAM J., LOOSLI J. K., MAYNARD L. A., 1954. Estimation of growth requirements for amino acids by assay of the carcass. J. biol. Chem., 208, 277-286.

WYLLIE D., OWEN J. B., 1978. Dietary protein content and the subsequent body composition and food intake of pigs. J. agric. Sci., Camb., 90, 69-76. 